\title{
La enseñanza del Holocausto: una oportunidad pedagógica para reflexionar sobre los conflictos sociales actuales
}

Teaching the Holocaust: a pedagogical opportunity to reflect on the present social conflicts

Guadalupe López Mateu*

\section{Resumen}

Tras una experiencia de campo, realizando visitas guiadas en un campo de concentración nazi y una intervención en las aulas de cuarto curso de Educación Secundaria, se han detectado unas problemáticas en la enseñanza del Holocausto que se anteponen a los objetivos de la misma: analizar lo que supuso apartar y exterminar a todo aquel que es "diferente", para reflexionar sobre las exclusiones y discriminaciones actuales. Para resolver dichas problemáticas, en este estudio se propone un enfoque alternativo al tratamiento del Holocausto en las aulas, en primer lugar, atendiendo debidamente a todos los colectivos que fueron víctimas de esta violencia potencial, sin establecer jerarquías en cuanto al grado de sufrimiento, y en segundo lugar, incidiendo en la responsabilidad que tenemos los individuos frente a estos actos de violencia e injusticias sociales, para así contribuir a la formación de una ciudadanía crítica, respetuosa y solidaria.

Palabras clave: Holocausto. Conflictos sociales actuales. Enseñanza de la historia. Responsabilidad social. Educación secundaria. Museos. Campos de concentración nazis.

\begin{abstract}
After a field experience on guided tours in a nazi concentration camp and an intervention in several fourth course secondary education lessons, some difficulties have been detected within the pedagogy of the Holocaust which are previous to the aims of this one: analyzing the marginalisation and the extermination of who was considered "different" in order to reflect on the current exclusions and discriminations. With the aim of solving those problems, it is proposed in this study an alternative approach to the treatment of the Holocaust in lectures: in the first place, paying due attention to those collectives which were victims of this potential violence, without the establishment of hierarchies regarding the level of suffering. Secondly, having an impact on the responsibility that we have in connection with these acts of violence and social injustices in order to contribute to the training of a critical, solidary and respectful citizenship.
\end{abstract}

Key words: Holocaust. Present social conflicts. History Education. Social responsibility. Secondary education. Museum. Nazi concentration camps.

*Universitat de València•gualoma@uv.es

López Mateu, G. (2020). La enseñanza del Holocausto: una oportunidad pedagógica para reflexionar sobre los conflictos sociales actuales. CLIO. History and History teaching, 46, 30-40. http://doi.org/10.26754/ojs clio/clio.2020465300. Recibido: 8/9/2020. Aceptado: 26/11/2020. 


\section{Introducción}

Desde la segunda mitad del siglo XX, se han creado diversas instituciones e iniciativas a nivel internacional que promueven planes de estudio sobre el Holocausto, con el objetivo de consolidar en el alumnado valores como la igualdad, la tolerancia y el respeto sin distinción de etnia, género, religión o lengua. $Y$ a su vez, aspiran a que el alumnado reflexione sobre las situaciones que conllevan a un riesgo de genocidio en el mundo contemporáneo, pues como apunta el historiador Yehuda Bauer (2012):

Tanto si se vive en África central, como en China, el Pacífico sur o Suiza, se tiene que ser consciente del peligro que representa el genocidio. A fin de cuentas, educar sobre el Holocausto significa en última instancia alejar lo máximo posible a la humanidad de esa forma extrema de asesinato masivo (p. 7).

Sin embargo, pese a los esfuerzos de muchos supervivientes, activistas e instituciones por difundir la memoria del Holocausto y concienciar a la población sobre los peligros que conlleva la intolerancia y el odio hacia el "otro", existen movimientos negacionistas y la violencia genocida se ha repetido con posterioridad a este hecho histórico. Además, cabe señalar que en Europa, están resurgiendo grupos políticos de extrema derecha con un gran apoyo por parte de la población, que rescatan aquellos discursos de odio que culminaron en el asesinato masivo de once millones de personas setenta y cinco años atrás.

Por lo tanto, es necesario cuestionarse cómo es posible que esto esté sucediendo en Europa si desde las dos últimas décadas el Holocausto está cada vez más presente en los planes de estudio de muchos países del continente. Así pues, en este estudio se plantea que, probablemente, la respuesta se halle en el tratamiento tradicional que se le da al Holocausto en el ámbito educativo, ya que tras unas observaciones realizadas en un museo dedicado a su difusión y una posterior intervención en un aula de Secundaria, se han detectado unas problemáticas (repetidas en ambos contextos), que se anteponen a los objetivos de la enseñanza del Holocausto: analizar lo que supuso apartar y exterminar a todo aquel que es "diferente", para reflexionar sobre las exclusiones y discriminaciones actuales. 
De tal manera que, a continuación se expondrán dichas problemáticas para posteriormente proponer un enfoque alternativo al tratamiento del Holocausto en las aulas, que cumpla con los objetivos de la enseñanza del mismo.

\section{Problemáticas detectadas en la enseñanza-aprendizaje del Holocausto}

Problemáticas detectadas en el tratamiento del Holocausto en un campo de concentración musealizado

Quien suscribe estas líneas, del año 2017 al 2019 trabajó realizando visitas guiadas en un campo de concentración nazi conocido como Theresienstadt, el cual se encuentra ubicado en el centro de Bohemia, Chequia, por lo que durante esta experiencia profesional, se detectaron tres problemáticas que se anteponían a las finalidades didácticas del Holocausto: la primera de ellas, en relación al discurso museográfico del campo de concentración; la segunda, en torno al tipo de conocimiento que poseían los visitantes sobre este acontecimiento histórico; y la tercera, sobre el enfoque e interpretación por parte de los visitantes sobre el Holocausto en relación a las conexiones con problemas sociales actuales.

En relación a la primera problemática, el campo de concentración de Theresienstadt cuenta con un Museo de la Memoria del Holocausto, dotado de todo tipo de recursos visuales y materiales para que los guías apoyen visualmente el discurso, no obstante, el discurso museográfico resulta bastante incompleto, ya que se centra exclusivamente en la historia de los judíos europeos en época contemporánea y el antisemitismo, sin atender al resto de víctimas ni exponer las diferentes formas de odio que se dieron durante el Holocausto, las cuales no han sido superadas por la sociedad actual. Por tal motivo, el equipo de guías al que pertenezco, consideramos imprescindible incluir en el discurso a todas las víctimas. Pero en ese instante, nos topamos con la segunda problemática, la mayoría de los visitantes, todos ellos hispanohablantes y en su mayoría procedentes de España, pero también de otros países de Centro y Sudamérica, no son conscientes de que a parte del colectivo judío, hubo otras minorías afectadas por el Holocausto, y más concretamente, desconocen por completo que entre las víctimas se encontraban ciudadanos de origen español, algo bastante significativo por parte de los visitantes españoles. 
Tal y como apuntan Valls, Parra y Fuertes (2017), este desconocimiento se debe a que en España todavía no se ha desarrollado una sólida política de memoria histórica que haga alusión y recuerde el sufrimiento de todos los ciudadanos que lucharon contra el franquismo y el nacionalsocialismo, aun siendo una pieza esencial en la instauración de la Democracia. Pues la muerte del dictador Franco no vino acompañada de la imprescindible revisión histórica y la reparación a las víctimas, por lo que se produjo una especie de "Democracia a cambio de olvido", donde se llevaron a cabo unas leyes de impunidad en lugar de leyes para la reconciliación. De tal manera que como afirman Valls et al (2017):

\footnotetext{
El tema de la reconciliación entre españoles respecto a su pasado es una cuestión casi totalmente ausente en los manuales escolares y dentro de las aulas. Ya que son temas muy complejos y delicados que dichos manuales no se atreven a enfrentar abiertamente como tampoco se atrevieron los partidos políticos (p.18).
}

Por tal motivo, no es de extrañar que, como se ha mencionado anteriormente, la mayoría de visitantes españoles consideren el Holocausto un acontecimiento histórico ajeno a su propia historia, algo que resulta un obstáculo para la ya mencionada y tan necesaria reconciliación de la sociedad española.

En cuanto a la tercera y última problemática, ésta está relacionada con la reacciones de los visitantes al establecer conexiones entre el Holocausto con conflictos sociales actuales. Pues, a lo largo de las visitas guiadas, incluimos en el discurso reflexiones sobre las marginaciones, exclusiones y odio hacia las minorías en nuestro días en comparación con las víctimas del Holocausto. Así pues, dichas comparaciones parten de lo cercano, ya que podríamos reflexionar sobre diversos conflictos sociales, pero apostamos por centrarnos en aquello que nos repercute de manera directa como ciudadanos, como por ejemplo las políticas de inmigración en Europa, así como la crisis de los refugiados o la islamofobia, para poder implicar directamente a los visitantes, ya que la mayoría de ellos provienen de Europa, y así fomentar la reflexión sobre la responsabilidad colectiva que todos tenemos ante estas complejas problemáticas sociales.

En cuanto a la reacción de los visitantes al tratar estas cuestiones, en algunas ocasiones resultan un tanto contradictorias, ya que a lo largo del recorrido, suelen mostrar una gran empatía con las víctimas del Holocausto, cuestionándose 
constantemente cómo pudo suceder algo así y por qué la sociedad europea de aquel momento lo permitió. Sin embargo, esta actitud suele cambiar cuando el guía compara, por ejemplo, fotografías en las que se muestran cadáveres en los campos de concentración nazis con otras en las que aparecen cadáveres de refugiados en las costas del Mediterráneo, ya que dicha comparación tiene como objetivo reflexionar sobre la responsabilidad social que tenemos ante las crisis humanitarias. Ante esta situación, gran parte de los visitantes se sienten incómodos, desconcertados o incluso atacados, pues el ejercicio de autocrítica y el asumir nuestra propia responsabilidad como espectadores o testigos (pasivos) genera una gran confrontación, y del mismo modo existen ciertos prejuicios basados en estereotipos muy difíciles de deconstruir, los cuales originan que aquella empatía inicial hacia las víctimas, se desvanezca por completo cuando se establecen comparaciones con las víctimas actuales.

Por lo tanto, al desarrollar el discurso sobre estos temas socialmente conflictivos, como es el Holocausto, tanto a través de los museos, las visitas guiadas y en las aulas, es imprescindible resaltar que la empatía turística o escolar con las víctimas no reemplaza la necesidad de que estos discursos contra-narrativos tengan objetivos explícitamente establecidos, como alentar a los turistas o a los estudiantes a comprender la importancia de la solidaridad con los oprimidos y perseguidos en el presente.

Problemáticas detectadas en las ideas previas sobre el Holocausto en alumnado de Secundaria

Tras las observaciones realizadas en las visitas guiadas en el campo de concentración, tuve la oportunidad de intervenir en un aula de $4^{\circ}$ de la ESO en la materia de Geografía e Historia, llevando a cabo una unidad didáctica dedicada íntegramente al Holocausto. De tal manera que aproveché la oportunidad para analizar si las problemáticas detectadas que se han citado en el apartado anterior se repetían en las aulas de Secundaria.

Para ello, inicié dicha unidad didáctica realizando un análisis sobre las ideas previas que poseía el alumnado acerca del Holocausto, a través de un cuestionario con tres preguntas que debían responder por escrito e individualmente en un corto periodo de 
tiempo, para posteriormente ponerlas en común de manera oral. Dichas preguntas fueron: 1) ¿Sabrías definir qué fue el holocausto?; 2) ¿Qué personas fueron víctimas del Holocausto?; 3) ¿De dónde has obtenido la información: familia/amigos; series/películas; videojuegos; redes sociales/Youtube/Wikipedia/Google; libros/revistas/cómics; otros?

El objetivo de estas preguntas fue: en primer lugar, analizar los conocimientos y las representaciones que el alumnado tenía sobre el Holocausto y comprobar si del mismo modo que los visitantes en los campos de concentración, el alumnado asociaba exclusivamente el Holocausto al genocidio del pueblo judío; y en segundo lugar, averiguar de dónde habían obtenido la información para poder contextualizar sus respuestas.

En cuanto a las respuestas del alumnado, a la primera pregunta, la mayoría de ellos contestaron de manera muy similar a las que se exponen a continuación:

"El Holocausto fue el genocidio masivo de los judíos" // "Un acontecimiento donde murió mucha gente" // "La época en que los nazis mataban a los judíos y mataron a millones de ellos" // "El exterminio de lo que se suponía que eran razas inferiores".

A la segunda pregunta en relación a quiénes fueron las víctimas del Holocausto, el $100 \%$ del alumnado respondió que fueron judíos. Mientras que una parte reducida mencionó además a otros colectivos, como por ejemplo: homosexuales (30\%); prisioneros políticos (26\%); negros (25\%); musulmanes (25\%); o gitanos (23\%). Cabe señalar que dos de los treinta alumnos hicieron mención a prisioneros de origen español y tan solo uno, hizo referencia a víctimas con diversidad funcional. Algunas respuestas fueron las siguientes:

"Fueron víctimas del Holocausto los judíos y personas de raza oscura" // "Negros, judíos y musulmanes" // "Los judíos y la gente que se opuso a la dictadura" // "Los judíos y las personas que no eran altas, rubias y con los ojos claros".

Finalmente, a la tercera pregunta, el 92\% del alumnado respondió que había obtenido la información a través de series, películas y documentales, muchos de ellos haciendo mención a películas como La vida es bella, La ladrona de libros o El niño con el pijama de rayas. Un 73\% también destacó que de sus familiares y amigos. Un 57\% de redes sociales, Youtube o búsquedas en Google. Un 53\% de libros, revistas o cómics, aquí 
varios alumnos hicieron mención al cómic Capitán América o al Diario de Anna Frank. Un $38 \%$ de videojuegos como Call of Duty WWII y finalmente un $11 \%$ de hizo referencia a museos.

Tras analizar estas respuestas, la conclusión que se puede extraer es que en el imaginario colectivo del alumnado, el concepto del Holocausto significa principalmente el genocidio del pueblo judío a manos del régimen nazi, motivado por cuestiones puramente xenófobas o racistas. Por lo tanto, tras analizar las respuestas de la pregunta número 3 , se puede advertir, que esta idea preconcebida del alumnado sobre el concepto "Holocausto", está fuertemente influenciada por la cultura audiovisual, en cuanto a la literatura y la cinematografía se refiere, ya que la mayoría de películas o novelas se centran en la historia de las víctimas judías como sería el caso de las citadas por el alumnado: La vida es bella, El niño con el pijama de rayas o El diario de Anna Frank. Así que no es de extrañar que los y las estudiantes identifiquen principalmente como víctimas de este genocidio al colectivo judío.

No obstante, un pequeño porcentaje a través de las respuestas también apuntó a prejuicios e intolerancia por cuestiones políticas o de orientación sexual, y a su vez una parte considerable del alumnado incluyó entre las víctimas al colectivo gitano así como al colectivo musulmán (este último de manera errónea), y también hicieron mención a personas de piel negra, que aun siendo perseguidas por los nazis a lo largo de toda la Europa ocupada, no fueron víctimas como tal de un programa sistemático para su eliminación, como es el caso de los judíos y otros grupos.

Por lo tanto, a través de estas respuestas se puede observar que el alumnado hizo una asociación de la ideología nacionalsocialista a la intolerancia por cuestiones vinculadas directamente a la inferioridad racial, cosa que en cierto modo es acertada, pero a su vez, quedarse únicamente con la idea de que el Holocausto fue un genocidio masivo por cuestiones racistas o xenófobas supone no ser consciente del verdadero alcance de esta persecución y violencia extrema, e impide reflexionar sobre las múltiples formas de odio hacia las minorías sociales. 


\section{Propuesta de un enfoque alternativo al tratamiento del Holocausto en las aulas de Secundaria}

Una vez analizadas las problemáticas en torno a la enseñanza del Holocausto que se han expuesto en el apartado anterior, a continuación se va a plantear una serie de ideas sobre cómo consideramos que debería enfocarse el tratamiento del Holocausto, concretamente, en el ámbito educativo español, ya que este tema se encuentra tanto en el currículum de la materia de Geografía e Historia en 4º de la ESO, como en la de Historia del Mundo contemporáneo de $1^{\circ}$ de Bachillerato.

En primer lugar, consideramos necesario que el Holocausto tenga una mayor presencia en los manuales escolares de ambas materias, ya que en la actualidad, aparece contextualizado dentro de una unidad didáctica dedicada a la Segunda Guerra Mundial de manera muy esquemática y resumida, por lo que este tema suele ser tratado muy superficialmente en las aulas. Así pues, debido a la relevancia educativa del mismo para la educación en valores como la que demanda actualmente nuestra sociedad, sería conveniente plantear una unidad didáctica dedicada exclusivamente al Holocausto, para así poder profundizar en los contenidos debidamente.

En segundo lugar, en cuanto al enfoque de los contenidos, éstos deberían partir del enfoque didáctico defendido por el ya citado historiador Yehuda Bauer (2006): la enseñanza del Holocausto se debe plantear como uno de los genocidios de la Humanidad, no limitando a la experiencia judía durante el Tercer Reich, aunque dicha experiencia haya sido la más compleja y singular hasta la fecha, algo que debe ocupar un espacio y un protagonismo propio, pero a su vez, implicaría desde una perspectiva curricular, atender en las clases de Geografía e Historia a otros casos de violencia genocida como el armenio o los de Ruanda y Camboya. Así pues, según la UNESCO (2013) la comparación del Holocausto (que no trivialización):

Facilita la comprensión de las características específicas y las diferencias entre los distintos acontecimientos estudiados. Sin embargo, aunque pedagógicamente sea valioso hacer un estudio comparativo de los genocidios, resulta muy importante no intentar hacer una comparación del sufrimiento y estudiar los eventos tomando siempre en cuenta su contexto histórico particular, [...] pues todos los ejemplos de violencia masiva, incluido el Holocausto, deben comprenderse en función de sus características singulares y su realidad no puede en ningún caso ser minimizada, trivializada o negada (p. 17). 
Por lo tanto, esta manera integrada de abordar el Holocausto, permitirá generar en el alumnado reflexiones más amplias sobre prácticas xenófobas, discriminatorias y antidemocráticas en diferentes contextos. Pero para ello, no solo bastará con la comparación de este genocidio con otros, sino con la debida atención a todas las víctimas del Holocausto, sin establecer jerarquías en cuanto al grado de sufrimiento de cada una de ellas, ni minusvalorar a ninguno de los colectivos que sufrieron los horrores perpetrados por los nacionalsocialista alemanes. En este sentido, se debe romper con el discurso tradicional, que focaliza de manera casi exclusiva el sufrimiento de las víctimas judías, ya que no atender debidamente la persecución, discriminación y asesinato masivo de otros colectivos como los romaní y sinti, los homosexuales, personas con diversidad funcional, opositores políticos y otros grupos religiosos, como los testigos de Jehová, supone legitimar un discurso segregador incompatible con los valores de solidaridad, justicia social, igualdad, respeto y tolerancia que precisamente promueven las instituciones dedicadas al estudio y la enseñanza del Holocausto (UNESCO, 2015) y a su vez, impide reflexionar sobre las discriminaciones y marginaciones que ciertas minorías sociales sufren en la actualidad.

Del mismo modo, en relación a las víctimas, consideramos especialmente relevante no pasar por alto en las aulas que la represión nazi también repercutió a miles de españoles exiliados tras la Guerra Civil, ya que sus historias han sido olvidadas a causa de la indiferencia dominante hacia los exiliados y deportados por parte de la mayoría de gobiernos que han representado el Estado español. Así pues, en las últimas dos décadas, muchos investigadores europeos han reivindicado la necesidad de incorporar la memoria histórica en el ámbito escolar, para evitar que la escritura de la historia oficial e institucionalizada sea manipulada, pues como afirman los historiadores Prats y Santacana (2011):

Hay olvidos que son usados para ciertos fines de ocultación de las represiones o de las dictaduras y, al tiempo, sirven a los que, de manera interesada, han defendido los perdones y amnistías que han seguido a los grandes crímenes al siglo XX, ahondando en lo que Ricoeur denomina el "olvido feliz", o la reconciliación de un pueblo con los grandes traumas colectivos de su historia ( $p$. 20)

De tal manera que, recuperar la memoria del Holocausto, así como la de las víctimas de los traumas históricos recientes tiene una clara función de saneamiento de las 
sociedades que los han sufrido, y es necesario que esto sea a través de la enseñanza escolar (Prats y Santacana, 2011).

Finalmente, también es necesario que a través de los contenidos, se incida en la importancia de la responsabilidad que tiene la sociedad hacia los conflictos sociales. En el caso concreto del Holocausto, sería conveniente analizar la responsabilidad que tuvo la sociedad europea durante la Segunda Guerra Mundial, como testigos directos del genocidio, teniendo en cuenta las diferentes posturas que adoptaron dichos testigos, y problematizando sobre aquellos que se decantaron por la pasividad hacia la intolerancia, el abuso o la violencia y por el contrario, poniendo en valor a los que defendieron a las minorías afectadas. Pues de este modo, el alumnado podrá establecer conexiones entre las persecuciones y la marginación de las víctimas del pasado con las del presente, y así podrá reflexionar sobre su propia responsabilidad ante los conflictos sociales y ser consciente de la necesidad de la toma de conciencia individual para no dejarse persuadir por políticas de odio, intolerancia y violencia, por muy adoctrinadoras que estas sean. Por lo tanto, reflexionar sobre la responsabilidad individual tiene una gran potencialidad didáctica para contribuir a la formación de un alumnado crítico, democrático y responsable.

\section{Conclusiones}

Por todo lo señalado a lo largo de este estudio, consideramos que el objetivo de la enseñanza del Holocausto, debe ser abordar su estudio desde una perspectiva que contribuya a que el alumnado sea capaz de establecer conexiones entre pasado y presente para dar respuesta a problemas socialmente relevantes que nos atañen en la actualidad. $\mathrm{Y}$ del mismo modo, debe incitar a la reflexión sobre comportamientos violentos como la xenofobia, el racismo, el odio o la indiferencia, entendiéndolos como hechos no exclusivos del Holocausto, pues como se ha expuesto en los párrafos anteriores la violencia genocida se ha ido repitiendo con posterioridad y recientemente podemos observar repuntes de violencia e intolerancia en muchas sociedades. De esta manera, la enseñanza del Holocausto partirá del compromiso con la actualidad, como un instrumento motivador del cambio social y político que contribuirá a la formación de una ciudadanía crítica, respetuosa y solidaria. 


\section{Referencias}

Alaize, B. (2009). L'ensenyament de la Shoah a l'escola francesa: reptes pedagògics i de memòria. En J. Pagès y M. González (Coords.), Història, memoria i ensenyament de la historia; perspectives europees i llatinoamericanes (pp. 69-81). Universitat Autònoma de Barcelona.

Bauer, Y. (2006). El Holocausto: Lo singular y lo universal. https://www.yadvashem.org/es/ education/educational-materials/lesson-plans/january-2006/yehuda-bauer.html

López Facal, R. y Santidrian, V. (2011). Los conflictos candentes en el aula. Iber. Didáctica de las Ciencias Sociales, Geografía e Historia, 69, 8-20.

Pagès, J. y Casas, M. (2005): Republicans i republicanes als camps de concentració nazis. Testimonis i recursos didàctics per a l'ensenyament secundari. Institut d'Educació de l'Ajuntament de Barcelona.

Prats, J. y Santacana, J. (2011). Didáctica de la Geografía y la Historia. En J. Prats (Coord.), Por qué y para qué enseñar Historia (pp. 13-27). GRAÓ.

Suarez Álvarez, M. (2012). La enseñanza del Holocausto en Bachillerato: Reflexiones y Propuestas. https://www.yadvashem.org/es/education/educational-materials/proposals/ reflections.html

UNESCO (2015). La Situación internacional de la enseñanza del Holocausto: panorama mundial en los manuales escolares y los planes de estudios, resumen. https://unesdoc.unesco.org/ark:/48223/pf0000233964 spa

UNESCO (2013). ¿Por qué enseñar sobre el Holocausto? http://cprtrujillo.juntaextremadura.net/public/Memoria SHOA/4\%20UNESCO\%20FOLLET O\%20HOLOCAUSTO.pdf

Valls, R., Parra, D. y Fuertes, C. (2017). Los temas históricos conflictivos y su abordaje escolar: un ejemplo español. Clío \& Asociados: La Historia Enseñada, 25, 8-21. https://doi.org/10.14409/cya.v0i25.6917 